\title{
A Population-Based Study Evaluating Family Physicians' HIV Experience and Care of People Living With HIV in Ontario
}

\author{
Claire E. Kendall, MD, $\mathrm{MSc}^{1-4}$ \\ Douglas G. Manuel, MD, MSc, \\ FRCPC ${ }^{1-3}$ \\ Jaime Younger, $M S c^{3}$ \\ William Hogg, MD, MSc, FRCPC, \\ CCFP $^{1,2}$
}

Richard H. Glazier, MD, MPH, CCFP, FCFP ${ }^{4-7}$

\author{
Monica Taljaard, $\mathrm{PbD}^{3,8}$ \\ ${ }^{1}$ C.T. Lamont Primary Health Care \\ Research Centre, Bruyère Research Insti- \\ tute, Ottawa, Ontario, Canada \\ ${ }^{2}$ Department of Family Medicine, Univer- \\ sity of Ottawa, Ottawa, Ontario, Canada \\ ${ }^{3}$ Ottawa Hospital Research Institute, \\ Ottawa, Ontario, Canada
}

${ }^{4}$ Institute for Clinical Evaluative Sciences, Toronto, Ontario, Canada

${ }^{5}$ Centre for Research on Inner City Health, Li Ka Shing Knowledge Institute, St. Michael's Hospital, Toronto, Ontario, Canada

${ }^{6}$ Department of Family and Community Medicine, St. Michael's Hospital, Toronto, Ontario, Canada

${ }^{7}$ Department of Family and Community Medicine, University of Toronto, Toronto, Ontario, Canada

${ }^{8}$ Department of Epidemiology and Community Medicine, University of Ottawa, Ottawa, Ontario, Canada

Conflicts of interest: authors report none.

\section{CORRESPONDING AUTHOR}

Claire E. Kendall, MD, MSc 43 Bruyère St, Floor 3JB Department of Family Medicine University of Ottawa Ottawa, Ontario, K1N 5C8 ckendall@uottawa.ca

\begin{abstract}
PURPOSE Greater physician experience managing human immunodeficiency virus (HIV) infection has been associated with better HIV-specific outcomes. The objective of this study was to evaluate whether the HIV experience of a family physician modifies the association between the model of care delivery and the quality of care for people living with HIV.
\end{abstract}

METHODS We retrospectively analyzed data from a population-based observational study conducted between April 1, 2009, and March 31, 2012. A total of 13,417 patients with HIV in Ontario were stratified into 5 possible patterns or models of care. We used multivariable hierarchical logistic regression analyses, adjusted for patient characteristics and pairwise comparisons, to evaluate the modification of the association between care model and indicators of quality of care (receipt of antiretroviral therapy, cancer screening, and health care use) by level of physician HIV experience $(\leq 5,6-49, \geq 50$ patients during study period).

RESULTS The majority of HIV-positive patients (52.8\%) saw family physicians exclusively for their care. Among these patients, receipt of antiretroviral therapy was significantly lower for those receiving care from family physicians with 5 or fewer patients and 6-49 patients compared with those with 50 or more patients (mean levels of adherence [95\% Cls] were 0.34 [0.30-0.39] and 0.40 [0.34-0.45], respectively, vs 0.77 [0.74-0.80]). Patients' receipt of cancer screenings and health care use were unrelated to family physician HIV experience.

CONCLUSIONS Family physician HIV experience was strongly associated with receipt of antiretroviral therapy by HIV-positive patients, especially among those seeing only family physicians for their care. Future work must determine the best models for integrating and delivering comprehensive HIV care among diverse populations and settings.

Ann Fam Med 2015;13:436-445. doi: 10.1370/afm.1822.

\section{INTRODUCTION}

A ntiretroviral therapy (ART) has substantially reduced the mortality and morbidity arising from human immunodeficiency virus (HIV) infection, thus shifting the care needs of people living with longstanding HIV. Evidence suggests that clinicians with more HIV training, HIV experience, or both provide higher quality of care as measured by disease-specific indicators, including ART prescribing. ${ }^{1-6}$ Many of these studies, however, were performed early in the ART era, when both disease and treatments were novel and complex. Recent work has found similar quality of HIV-specific care between generalist and specialist physicians, even though the HIV experience of these physicians varied. ${ }^{7,8}$ In addition, multidisciplinary care teams with nonexpert family physicians may improve patients' ART adherence more than HIV specialist care alone. ${ }^{9}$

Another important consideration is that specialist HIV physicians are less adept than generalists at preventing and managing the common comorbidities emerging with increased HIV longevity. ${ }^{10-18}$ There is 
increasing recognition that the management of HIV requires a primary care presence, but we have a poor understanding of how to integrate HIV-specific expertise within a primary care context. ${ }^{7,10,14-21}$ Overall, it has been difficult to distinguish the respective contributions of physician qualification and physician experience of care to the effectiveness of HIV care. ${ }^{3}$

We have previously used routinely collected administrative data to develop and characterize an intuitive typology of shared care for people with HIV based on actual patterns of care. ${ }^{22,23}$ We found that most HIV patients were linked to a usual family physician, and few saw HIV specialists exclusively. We further found that how care was delivered had a strong impact on the quality of care provided, with models of care including family physicians associated with better adherence to preventive interventions and fewer hospitalizations, and models including HIV specialists associated with better adherence to ART prescribing. ${ }^{24}$

The objective of this study was to explore whether the HIV experience of the family physician modifies the association between the model of care delivery and the quality of care for people living with HIV. We hypothesized that ART prescribing would be modified by the HIV experience of the family physician, but that routine cancer screening and health care use would not.

\section{METHODS}

\section{Data Sources}

We used the administrative databases held at the Institute for Clinical Evaluative Sciences (ICES) for this study. These databases are made available to accredited researchers through a data-sharing agreement with the Ontario Ministry of Health and Long-Term Care and are linked using unique, encoded identifiers. We specifically used the Registered Persons Database, which includes demographic and mortality data for all residents eligible for provincial health care 2006 Statistics Canada census data to link postal code of residence to attribute the household income quintile as a proxy for socioeconomic status; the Ontario Health Insurance Plan (OHIP) billing claims system, which contains $95 \%$ of physician services provided in the province; the Discharge Abstract Database, which captures all provincial hospital admission discharge data; the National Ambulatory Care Reporting System, which contains information on emergency department visits; Citizen and Immigration Canada data, which contains information on individuals granted permanent residency in Canada; the Client Agency Program Enrolment registry, which tracks patient enrollment to individual family physicians; the
ICES Physician Database, which is derived from the OHIP Corporate Provider Database, the Ontario Physician Human Resources Data Centre database, and the OHIP database of physician billings, and which contains information on physician demographics, training, and practice setting; and the Ontario Drug Benefits, a claims database of prescriptions to individuals covered by the public system, including those aged 65 years and older and those receiving social assistance (Ontario Works, Ontario Disability Support Program), or eligible for the subsidized catastrophic access Trillium program.

\section{Eligible Population}

Using OHIP billing claims, we applied a previously validated algorithm ${ }^{25}$ to people aged 18 years and older and living in Ontario between April 1, 1992, and March 31,2012 with a sensitivity of $96.2 \%$ (95\% CI, 95.2\%$97.9 \%)$ and specificity of $99.6 \%$ (95\% CI, 99.1\%-99.8\%) for identifying people with HIV receiving care in Ontario. We excluded patients with an invalid or outof-province residence on July 1, 2009 (277 patients). We excluded those who were known to be receiving care in community health centers between 2008 to 2010 (17 patients), as physicians in these centers are salaried and do not submit billing to OHIP. ${ }^{26}$ To avoid misclassifying their model of care delivery based on censored visit pattern data, we excluded patients who died during the 3-year study period (510 patients).

\section{Assignment of Patients to a Model of Care Delivery}

We used the OHIP database to identify all outpatient physician visits made by our cohort of HIV patients between April 1, 2009, and March 31, 2012. We then used a previously reported approach to assign patients to 1 of 5 models of care delivery: exclusively primary care, family physician-dominant comanagement (family physician has the most visits for HIV care), specialist-dominant comanagement (HIV specialist has the most visits for HIV care), exclusively HIVspecialist care, and low engagement. ${ }^{23}$ Briefly, physician specialty was categorized into 4 groups: family medicine (comprising the specialties of family medicine, family medicine/emergency medicine, general practice, or community medicine, all of whom may be licensed to practice family medicine/general practice in Ontario), internal medicine, infectious diseases, or other. In contrast to the United States, where internal medicine specialists may act as primary care clinicians, Canadian internal medicine specialists are primarily consultant physicians. Physicians were deemed to be HIV specialists if they were infectious disease or internal medicine specialists who had HIV-specific outpa- 
Figure 1. Flow diagram of study participants.

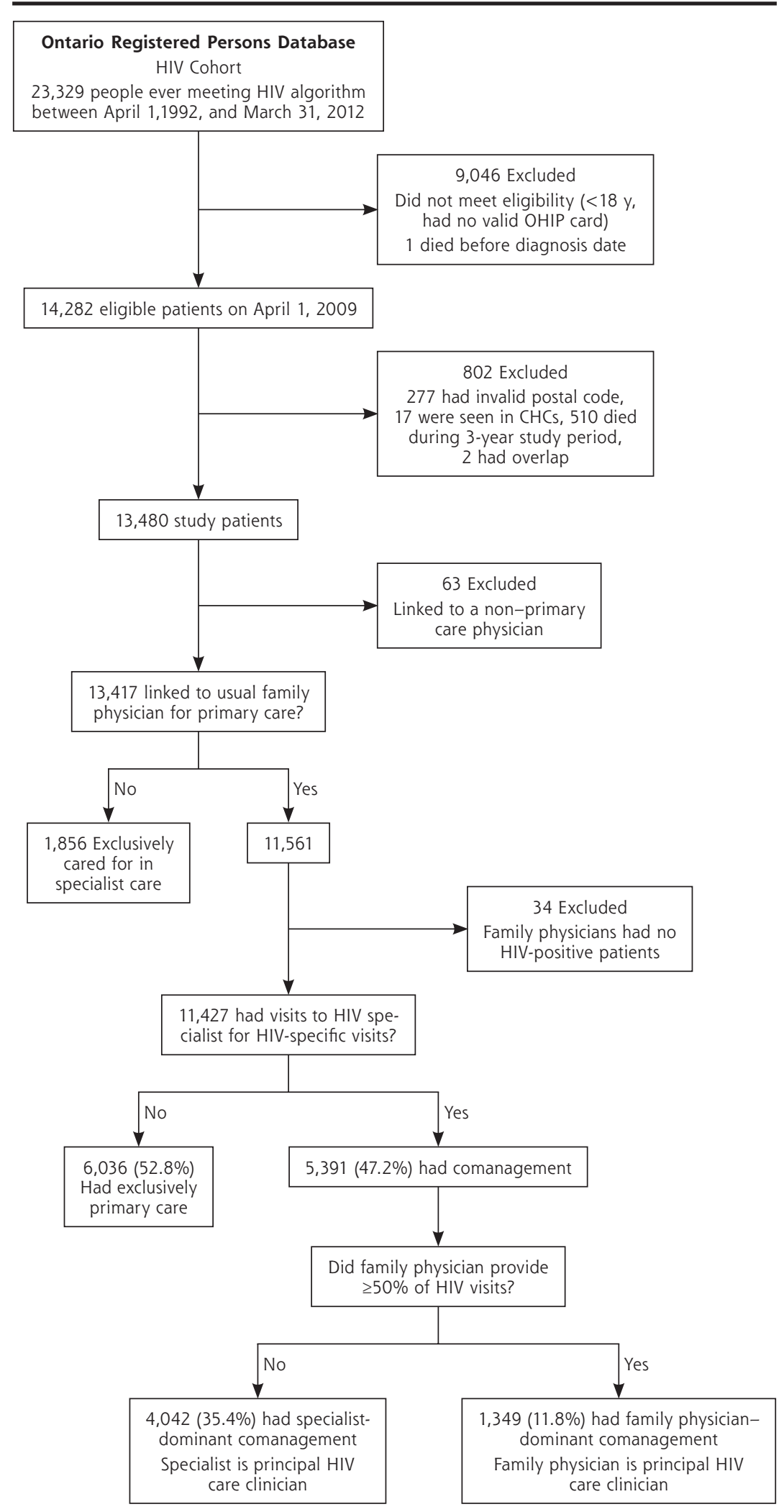

$\mathrm{CHC}=$ community health center; HIV = human immunodeficiency virus; OHIP = Ontario Health Insurance Plan. tient billing claims to patients in our HIV cohort. Patients were then assigned to models based on their visit data, including the specialty of the physician seen-either family physician or HIV specialist-and the billing codes submitted-either HIV-related (International Classification of Diseases, 9th Revision diagnosis code for visit was 042, 043, or 044) or non-HIVrelated (Figure 1). Patients who were contractually assigned to a family physician but who had no visits to that physician during the study period were excluded from analyses. Our main analyses were restricted to the 3 models of care delivery in which patients had a usual family physician.

\section{Patient Characteristics}

Patient age, sex, and postal code on April 1, 2009, were obtained from the Registered Persons Database. We used neighborhood-level postal codes linked to 2006 Statistics Canada census data to assign income quintiles and rurality scores: major urban areas (score 0 to 9 ), non-major urban areas (10 to 39), and rural areas $(\geq 40) .{ }^{27}$ We used data from Citizenship and Immigration Canada to categorize patient immigration status as follows: Canadian born, immigrant from European or western nations, immigrant from Africa or the Caribbean, and immigrant from other country. Immigrants from the HIV-endemic regions of Africa and the Caribbean were subcategorized here as they represent a growing proportion of new and prevalent HIV infections in Canada, are often demographically different from other groups, and have health outcomes that vary from those of others living with HIV. ${ }^{28-32}$ 
The Johns Hopkins Adjusted Clinical Group System was used to ascertain comorbidity by assigning patients to up to 32 distinct aggregated diagnosis groups (ADGs), and categorized patients as having a low $(\leq 5)$, medium (6 to 9$)$, or high $(\geq 10)$ number of ADGs. ${ }^{33,34}$ We used a previously validated method to identify individuals with mental health conditions. ${ }^{35}$ We used the Ontario Drug Benefits claims database to identify individuals who were eligible for public drug coverage and those who had actual claims during the study period.

\section{Family Physician HIV Experience}

We used the number of unique patients in the HIV cohort that a physician billed for at least once during the 3-year study period to determine the physician's experience in managing HIV, categorized as low ( $\leq 5$ patients), medium ( 6 to 49 patients), or high ( $\geq 50$ patients) expertise. We used these values as 6 to 49 patients is an HIV volume threshold that has been identified as possibly leading to better care and decreased mortality. ${ }^{2}$

\section{Outcome Measures}

Our primary outcome of interest was the receipt of any ART prescription among those eligible for Ontario drug benefits. Secondary outcomes included measures of preventive care: adherence to colorectal cancer screening (ascertained as 1 test over 2 years for individuals aged 50 to 74 years), adherence to breast cancer screening (ascertained as 1 mammography test over 2 years for women aged 50 to 69 years), and adherence to cervical cancer screening (ascertained as 1 cervical screening test over 2 years for women aged 21 to 69 years). They also included health service use outcomes: any emergency department visit over the latter 18 months of the 3-year study period (October 1, 2010, to March 31, 2012), and any hospital admissions (excluding maternity and same-day surgery). ${ }^{36}$

\section{Statistical Analysis}

We generated summary descriptive statistics to compare patient characteristics and outcomes among the 3 family physician HIV experience categories. As per ICES privacy requirements, cell sizes of 5 or fewer individuals are not reported.

We conducted multivariable hierarchical logistic regression analyses to examine the hypothesized associations with each quality outcome. We included main effects for model of care delivery and family physician HIV experience (modeled as a 3-level categorical variable), as well as the interaction between model and family physician HIV experience. We adjusted for patient characteristics: age, sex, neighborhood income quintile, rurality category, immigrant status category, level of comorbidity (ADG category), and presence of mental health conditions as fixed covariates in the model. We accounted for clustering by family physician using random effects. For each analysis, we determined the adjusted odds ratios (ORs) and obtained the predicted (least square) mean levels for each level of experience and within each model of care delivery, together with 95\% confidence intervals. Pairwise comparisons were conducted within each model of care delivery using the Tukey-Kramer adjustment for multiplicity. An overall level of significance of $5 \%$ was used. We conducted all statistical analyses using SAS version 9.3 (SAS Institute).

\section{Ethics Approval}

This study was approved by the Ottawa Hospital and Sunnybrook Health Sciences Centre Research Ethics Boards.

\section{RESULTS}

We identified 13,417 HIV-positive patients having a link to a usual family physician for primary care (Figure 1). The comparison of patient characteristics across models of care delivery has been described previously. ${ }^{24}$ Among the 11,427 patients meeting inclusion criteria, $52.8 \%$ were exclusively managed in primary care, whereas $47.2 \%$ had comanagement by a family physician and specialist. Categorized another way, $27.1 \%$ of patients had a family physician with a low level of HIV experience, $17.4 \%$ had one with a medium level, and $55.5 \%$ had one with a high level.

Table 1 shows the patient characteristics among different levels of family physician HIV experience. Patients seeing the most HIV-experienced family physicians were significantly more often male, urban dwelling, and Canadian born, and had lower comorbidity and a higher mean number of outpatient visits to their family physician during the study period. Although the observed prevalence of cancer screening varied among the experience levels, patients of family physicians with the highest level of experience had the highest observed prevalence of receipt of ART among eligible patients, as well as the lowest observed prevalences of any emergency department visit and any hospital admission.

Table 2 shows the results of the hierarchical logistic regression analyses for all study outcomes. Model of care delivery, family physician experience, and the interaction between these 2 variables were significantly associated with receipt of ART after adjusting for covariates. Model of care delivery was associated with receipt of colorectal cancer screening and any hospital 
Table 1. Characteristics of Patients Seen by Family Physicians Having Different Levels of HIV Experience

\begin{tabular}{|c|c|c|c|c|}
\hline Characteristic & $\begin{array}{l}\leq 5 \text { HIV Patients } \\
(n=3,098)\end{array}$ & $\begin{array}{l}\text { 6-49 HIV Patients } \\
(n=1,987)\end{array}$ & $\begin{array}{l}\geq 50 \text { HIV Patients } \\
(n=6,342)\end{array}$ & $\begin{array}{c}\text { Total } \\
(\mathrm{N}=11,427)\end{array}$ \\
\hline \multicolumn{5}{|l|}{ Patient characteristics } \\
\hline Male sex, No. (\%) & $2,066(66.7)$ & $1,388(69.9)$ & $5,786(91.2)$ & $9,240(80.9)$ \\
\hline Age, mean (SD), y & $45.8(12.0)$ & $44.7(10.6)$ & $45.0(9.6)$ & $45.2(10.5)$ \\
\hline \multicolumn{5}{|l|}{ Neighborhood income quintile, No. (\%) } \\
\hline Quintile 1 (lowest) & $938(30.3)$ & $787(39.6)$ & $1,827(28.8)$ & $3,552(31.1)$ \\
\hline Quintile 2 & $680(21.9)$ & $443(22.3)$ & $1,222(19.3)$ & $2,345(20.5)$ \\
\hline Quintile 3 & $508(16.4)$ & $290(14.6)$ & $1,061(16.7)$ & $1,859(16.3)$ \\
\hline Quintile 4 & $484(15.6)$ & $251(12.6)$ & $981(15.5)$ & $1,716(15.0)$ \\
\hline Quintile 5 (highest) & $475(15.3)$ & $204(10.3)$ & $1,174(18.5)$ & $1,853(16.2)$ \\
\hline Missing & $13(0.4)$ & $12(0.6)$ & $77(1.2)$ & $102(0.9)$ \\
\hline \multicolumn{5}{|l|}{ Rurality index, No. (\%) } \\
\hline Major urban & $2,437(78.7)$ & $1,835(92.4)$ & $6,042(95.3)$ & $10,314(90.3)$ \\
\hline Non-major urban & $527(17.0)$ & $122(6.1)$ & $244(3.8)$ & $893(7.8)$ \\
\hline Rural & $114(3.7)$ & $24(1.2)$ & $50(0.8)$ & $188(1.6)$ \\
\hline Missing & $20(0.6)$ & $6(0.3)$ & $6(0.1)$ & $32(0.3)$ \\
\hline \multicolumn{5}{|l|}{ Immigrant status, No. (\%) } \\
\hline Canadian born & $2,493(80.5)$ & $1,371(69.0)$ & $5,325(84.0)$ & $9,189(80.4)$ \\
\hline Immigrant from Africa or Caribbean & $327(10.6)$ & $432(21.7)$ & $474(7.5)$ & $1,233(10.8)$ \\
\hline $\begin{array}{l}\text { Immigrant from Europe or western } \\
\text { nations }\end{array}$ & $55(1.8)$ & $28(1.4)$ & $143(2.3)$ & $226(2.0)$ \\
\hline Immigrant from other nations & $223(7.2)$ & $156(7.9)$ & $400(6.3)$ & $779(6.8)$ \\
\hline Mental health condition, No. (\%) & $1,302(42.0)$ & $850(42.8)$ & $2,555(40.3)$ & $4,707(41.2)$ \\
\hline \multicolumn{5}{|l|}{ Comorbidity: ADG group, No. (\%) } \\
\hline High & $867(28.0)$ & $502(25.3)$ & $1,393(22.0)$ & $2,762(24.2)$ \\
\hline Medium & $1,142(36.9)$ & $687(34.6)$ & $2,159(34.0)$ & $3,988(34.9)$ \\
\hline Low & $1,089(35.2)$ & $798(40.2)$ & $2,790(44.0)$ & $4,677(40.9)$ \\
\hline Outpatient visits, mean (SD), No. & $14.9(15.2)$ & $16.2(21.6)$ & $17.3(13.8)$ & $16.4(15.8)$ \\
\hline \multicolumn{5}{|l|}{ Quality indicators, No. (\%) } \\
\hline Any ART prescription $(n=7,465)$ & $1,402(70.8)$ & $1,037(75.3)$ & $3,324(80.9)$ & $5,763(77.2)$ \\
\hline Colorectal cancer screening $(n=2,296)$ & $260(35.9)$ & $140(30.7)$ & $505(45.3)$ & $905(39.4)$ \\
\hline Cervical cancer screening $(n=1,946)$ & $446(50.7)$ & $225(41.1)$ & $268(51.5)$ & $939(48.3)$ \\
\hline Breast cancer screening $(n=513)$ & $148(54.8)$ & $73(57.0)$ & $54(47.0)$ & $275(53.6)$ \\
\hline Any emergency department visit & $1,117(36.1)$ & $685(34.5)$ & $1,910(30.1)$ & $3,712(32.5)$ \\
\hline Any hospital admission & $372(12.0)$ & $212(10.7)$ & $579(9.1)$ & $1,163(10.2)$ \\
\hline
\end{tabular}

admission, but neither family physician HIV experience nor the interaction term of model of care delivery and family physician HIV experience was significantly associated with these secondary outcomes.

Figure 2 displays the adjusted least square mean estimates of the probability of adherence to ART prescribing for each category of model of care delivery and family physician HIV experience. Among patients cared for exclusively in primary care, receipt of ART was significantly lower among those receiving care from family physicians with 5 or fewer patients and 6 to 49 patients compared with those receiving care from family physicians with 50 or more patients; the mean ART adherence (95\% CI) was 0.34 (0.30-0.39) and $0.40(0.34-0.45)$, respectively, vs 0.77 (0.74-0.80).
There were no statistically significant pairwise differences in receipt of ART by family physician experience within the other models of care delivery.

Table 3 shows the adjusted least square mean estimates of adherence for secondary outcomes. After adjustment for multiplicity, no pairwise comparisons in adherence for secondary outcomes were significant, indicating that family physician experience did not modify the effect for these outcomes.

\section{DISCUSSION}

We found that the model of care delivery (relative amounts of HIV specialist and family physician care) is important in meeting the evolving care needs of people 
Table 2. Hierarchical Logistic Regression Analysis of Receipt of ART, Cancer Screening, and Health Care Use

\begin{tabular}{|c|c|c|c|c|c|c|}
\hline Variable & $\begin{array}{c}\text { Receipt } \\
\text { of ART } \\
\text { AOR }(95 \% \mathrm{Cl})^{\mathrm{a}}\end{array}$ & $\begin{array}{c}\text { Colorectal } \\
\text { Cancer } \\
\text { Screening } \\
\text { AOR }(95 \% \mathrm{Cl})^{\mathrm{a}}\end{array}$ & $\begin{array}{c}\text { Cervical } \\
\text { Cancer } \\
\text { Screening } \\
\text { AOR }(95 \% \mathrm{Cl})^{\mathrm{a}}\end{array}$ & $\begin{array}{l}\text { Breast Cancer } \\
\text { Screening } \\
\text { AOR }(95 \% \mathrm{Cl})^{\mathrm{a}}\end{array}$ & $\begin{array}{c}\text { Any ED } \\
\text { Visit } \\
\text { AOR }(95 \% \mathrm{Cl})^{\mathrm{a}}\end{array}$ & $\begin{array}{c}\text { Any } \\
\text { Hospital } \\
\text { Admission }\end{array}$ \\
\hline \multicolumn{7}{|l|}{ Model of care delivery } \\
\hline $\begin{array}{l}\text { FP-dominant } \\
\text { comanagement }\end{array}$ & $\begin{array}{c}2.04 \\
(1.63-2.25)\end{array}$ & $\begin{array}{c}1.25 \\
(0.88-1.76)\end{array}$ & $\begin{array}{c}0.77 \\
(0.47-1.27)\end{array}$ & $\begin{array}{c}3.90 \\
(1.16-13.09)\end{array}$ & $\begin{array}{c}1.11 \\
(0.96-1.29)\end{array}$ & $\begin{array}{c}2.16 \\
(1.75-2.67)\end{array}$ \\
\hline $\begin{array}{l}\text { Specialist-dominant } \\
\text { comanagement }\end{array}$ & $\begin{array}{c}2.23 \\
(1.71-2.91)\end{array}$ & $\begin{array}{c}0.88 \\
(0.59-1.31)\end{array}$ & $\begin{array}{c}0.72 \\
(0.44-1.18\end{array}$ & $\begin{array}{c}0.49 \\
(0.13-1.79)\end{array}$ & $\begin{array}{c}1.11 \\
(0.94-1.31)\end{array}$ & $\begin{array}{c}1.57 \\
(1.22-2.02)\end{array}$ \\
\hline Exclusively primary care (ref) & $1.00^{\mathrm{b}}$ & $1.00^{\mathrm{b}}$ & 1.00 & 1.00 & 1.00 & $1.00^{\mathrm{b}}$ \\
\hline \multicolumn{7}{|l|}{ Family physician HIV experience ${ }^{b}$} \\
\hline$\leq 5$ HIV patients & $\begin{array}{c}0.16 \\
(0.12-0.21)\end{array}$ & $\begin{array}{c}0.85 \\
(0.56-1.29)\end{array}$ & $\begin{array}{c}1.09 \\
(0.70-1.69)\end{array}$ & $\begin{array}{c}2.10 \\
(0.84-5.27)\end{array}$ & $\begin{array}{c}1.16 \\
(0.98-1.37)\end{array}$ & $\begin{array}{c}1.17 \\
(0.89-1.53)\end{array}$ \\
\hline 6-49 HIV patients & $\begin{array}{c}0.20 \\
(0.15-0.27)\end{array}$ & $\begin{array}{c}0.67 \\
(0.42-1.08)\end{array}$ & $\begin{array}{c}0.59 \\
(0.35-0.99)\end{array}$ & $\begin{array}{c}1.69 \\
(0.58-4.91)\end{array}$ & $\begin{array}{c}1.12 \\
(0.92-1.37)\end{array}$ & $\begin{array}{c}1.40 \\
(1.02-1.91)\end{array}$ \\
\hline$\geq 50$ HIV patients (ref) & 1.00 & 1.00 & 1.00 & 1.00 & 1.00 & 1.00 \\
\hline \multicolumn{7}{|c|}{ Interaction modelc FP HIV experience } \\
\hline $\begin{array}{l}\text { FP-dominant comanage- } \\
\text { ment }^{c} \leq 5 \text { HIV patients }\end{array}$ & $\begin{array}{c}6.27 \\
(2.40-16.34)^{b}\end{array}$ & $\begin{array}{c}0.98 \\
(0.26-3.63)\end{array}$ & $\begin{array}{c}1.45 \\
(0.34-6.15)\end{array}$ & $\begin{array}{c}0.08 \\
(0.01-1.05)\end{array}$ & $\begin{array}{c}0.65 \\
(0.33-1.29)\end{array}$ & $\begin{array}{c}1.24 \\
(0.58-2.66)\end{array}$ \\
\hline $\begin{array}{l}\text { FP-dominant comanage- } \\
\text { ment } 6-49 \text { HIV patients }\end{array}$ & $\begin{array}{c}6.33 \\
(2.92-13.71)^{\mathrm{b}}\end{array}$ & $\begin{array}{c}1.17 \\
(0.44-3.07)\end{array}$ & $\begin{array}{c}0.67 \\
(0.18-2.51)\end{array}$ & $\begin{array}{c}0.09 \\
(0.01-1.08)\end{array}$ & $\begin{array}{c}0.85 \\
(0.53-1.36)\end{array}$ & $\begin{array}{c}0.70 \\
(0.38-1.30)\end{array}$ \\
\hline $\begin{array}{l}\text { SP-dominant comanage- } \\
\text { ment }^{c} \leq 5 \text { HIV patients }\end{array}$ & $\begin{array}{c}5.92 \\
(4.10-8.57)^{b}\end{array}$ & $\begin{array}{c}0.76 \\
(0.44-1.30)\end{array}$ & $\begin{array}{c}0.96 \\
(0.54-1.71)\end{array}$ & $\begin{array}{c}0.87 \\
(0.21-3.67)\end{array}$ & $\begin{array}{c}0.90 \\
(0.72-1.14)\end{array}$ & $\begin{array}{c}0.87 \\
(0.61-1.24)\end{array}$ \\
\hline $\begin{array}{l}\text { SP dominant comanage- } \\
\text { ment }^{\mathrm{C}} 6-49 \text { HIV patients }\end{array}$ & $\begin{array}{c}4.54 \\
(3.01-6.86)^{b}\end{array}$ & $\begin{array}{c}0.73 \\
(0.39-1.38)\end{array}$ & $\begin{array}{c}1.30 \\
(0.67-2.53)\end{array}$ & $\begin{array}{c}2.06 \\
(0.41-10.36)\end{array}$ & $\begin{array}{c}1.01 \\
(0.77-1.32)\end{array}$ & $\begin{array}{c}0.65 \\
(0.43-0.98)\end{array}$ \\
\hline Age, years & $\begin{array}{c}1.01 \\
(1.00-1.01)\end{array}$ & $\begin{array}{c}1.02 \\
(1.00-1.04)^{b}\end{array}$ & $\begin{array}{c}0.98 \\
(0.97-0.99)^{b}\end{array}$ & $\begin{array}{c}1.07 \\
(1.01-1.12)^{b}\end{array}$ & $\begin{array}{c}0.99 \\
(0.98-0.99)^{b}\end{array}$ & $\begin{array}{c}1.02 \\
(1.02-1.03)^{b}\end{array}$ \\
\hline Sex, female & $\begin{array}{c}0.88 \\
(0.74-1.04)\end{array}$ & $\begin{array}{c}1.05 \\
(0.79-1.39)\end{array}$ & - & - & $\begin{array}{c}1.07 \\
(0.95-1.20)\end{array}$ & $\begin{array}{c}0.97 \\
(0.82-1.16)\end{array}$ \\
\hline \multicolumn{7}{|l|}{ Neighborhood income quintile } \\
\hline Quintile 1 (lowest) & $\begin{array}{c}1.42 \\
(1.12-1.73)\end{array}$ & $\begin{array}{c}0.95 \\
(0.71-1.27)\end{array}$ & $\begin{array}{c}0.95 \\
(0.66-1.36)\end{array}$ & $\begin{array}{c}0.45 \\
(0.20-1.00)\end{array}$ & $\begin{array}{c}1.15 \\
(1.01-1.30)\end{array}$ & $\begin{array}{c}1.12 \\
(0.92-1.36)\end{array}$ \\
\hline Quintile 2 & $\begin{array}{c}1.05 \\
(0.85-1.28)\end{array}$ & $\begin{array}{c}0.88 \\
(0.64-1.21)\end{array}$ & $\begin{array}{c}0.98 \\
(0.66-1.44)\end{array}$ & $\begin{array}{c}0.32 \\
(0.14-0.76)\end{array}$ & $\begin{array}{c}1.00 \\
(0.87-1.15)\end{array}$ & $\begin{array}{c}1.02 \\
(0.83-1.27)\end{array}$ \\
\hline Quintile 3 & $\begin{array}{c}0.96 \\
(0.81-1.25)\end{array}$ & $\begin{array}{c}1.15 \\
(0.83-1.59)\end{array}$ & $\begin{array}{c}1.18 \\
(0.78-1.77)\end{array}$ & $\begin{array}{c}0.79 \\
(0.33-1.90)\end{array}$ & $\begin{array}{c}0.95 \\
(0.82-1.10)\end{array}$ & $\begin{array}{c}0.96 \\
(0.77-1.20)\end{array}$ \\
\hline Quintile 4 & $\begin{array}{c}1.09 \\
(0.88-1.34)\end{array}$ & $\begin{array}{c}0.86 \\
(0.62-1.21)\end{array}$ & $\begin{array}{c}1.09 \\
(0.71-1.66)\end{array}$ & $\begin{array}{c}0.68 \\
(0.27-1.76)\end{array}$ & $\begin{array}{c}0.90 \\
(0.77-1.04)\end{array}$ & $\begin{array}{c}0.88 \\
(0.69-1.11)\end{array}$ \\
\hline Quintile 5 (highest) (ref) & $1.00^{b}$ & 1.00 & 1.00 & $1.00^{d}$ & $1.00^{\mathrm{b}}$ & 1.00 \\
\hline \multicolumn{7}{|l|}{ Rurality index ${ }^{d}$} \\
\hline Non-major urban & $\begin{array}{c}1.35 \\
(1.04-1.69)\end{array}$ & $\begin{array}{c}0.95 \\
(0.68-1.32)\end{array}$ & $\begin{array}{c}0.91 \\
(0.61-1.35)\end{array}$ & $\begin{array}{c}0.54 \\
(0.25-1.15)\end{array}$ & $\begin{array}{c}1.49 \\
(1.28-1.73)\end{array}$ & $\begin{array}{c}1.41 \\
(1.13-1.75)\end{array}$ \\
\hline Rural & $\begin{array}{c}1.18 \\
(0.75-1.88)\end{array}$ & $\begin{array}{c}0.66 \\
(0.34-1.30)\end{array}$ & $\begin{array}{c}0.70 \\
(0.31-1.58)\end{array}$ & $\begin{array}{c}0.38 \\
(0.10-1.54)\end{array}$ & $\begin{array}{c}1.79 \\
(1.31-2.44)\end{array}$ & $\begin{array}{c}1.82 \\
(1.22-2.70)\end{array}$ \\
\hline Urban (ref) & 1.00 & 1.00 & 1.00 & 1.00 & $1.00^{\mathrm{b}}$ & $\begin{array}{l}1.00^{\mathrm{b}} \\
\text { continues }\end{array}$ \\
\hline
\end{tabular}

with HIV, and additionally, that the HIV experience of family physicians modifies the association between model of care delivery and quality of care outcomes for HIV-related care. In particular, we found that family physician HIV experience was strongly associated with receipt of ART by HIV-positive patients, especially among those seeing only family physicians for their care ; those seeing a family physician with the highest level of HIV experience were almost twice as likely to receive ART as counterparts seeing less experienced family physicians. In contrast, cancer screening and health service use were not influenced by family physician HIV experience. Given the need to shift HIV care to community-based models of care delivery, this study teases out the respective impacts of physician specialty and physician HIV experience by demonstrating that the most comprehensive care is delivered by family physicians with high HIV experience.

Our findings are consistent with those of a previous systematic review demonstrating that physician HIV experience is specifically associated with improved HIV-specific outcomes, such as adherence to ART prescribing. ${ }^{2}$ Another review conducted by the same authors ${ }^{3}$ attempted to distinguish physician training 
Table 2. Hierarchical Logistic Regression Analysis of Receipt of ART, Cancer Screening, and Health Care Use (continued)

\begin{tabular}{|c|c|c|c|c|c|c|}
\hline Variable & $\begin{array}{c}\text { Receipt } \\
\text { of ART } \\
\text { AOR }(95 \% \mathrm{Cl})^{a}\end{array}$ & $\begin{array}{l}\text { Colorectal } \\
\text { Cancer } \\
\text { Screening } \\
\text { AOR }(95 \% \mathrm{CI})^{\mathrm{a}}\end{array}$ & $\begin{array}{l}\text { Cervical } \\
\text { Cancer } \\
\text { Screening } \\
\text { AOR }(95 \% \mathrm{Cl})^{\mathrm{a}}\end{array}$ & $\begin{array}{l}\text { Breast Cancer } \\
\text { Screening } \\
\text { AOR }(95 \% \mathrm{CI})^{\text {a }}\end{array}$ & $\begin{array}{c}\text { Any ED } \\
\text { Visit } \\
\text { AOR }(95 \% \mathrm{Cl})^{\mathrm{a}}\end{array}$ & $\begin{array}{l}\text { Any } \\
\text { Hospital } \\
\text { Admission }\end{array}$ \\
\hline \multicolumn{7}{|l|}{ Immigrant status ${ }^{b}$} \\
\hline $\begin{array}{l}\text { Immigrant from Africa or } \\
\text { Caribbean }\end{array}$ & $\begin{array}{c}1.58 \\
(1.24-1.99)\end{array}$ & $\begin{array}{c}0.90 \\
(0.60-1.34)\end{array}$ & $\begin{array}{c}1.04 \\
(0.81-1.33)\end{array}$ & $\begin{array}{c}1.66 \\
(0.90-3.09)\end{array}$ & $\begin{array}{c}0.77 \\
(0.67-0.90)^{b}\end{array}$ & $\begin{array}{c}0.80 \\
(0.63-1.02)^{b}\end{array}$ \\
\hline $\begin{array}{l}\text { Immigrant from Europe or } \\
\text { western nations }\end{array}$ & $\begin{array}{c}0.78 \\
(0.50-1.24)\end{array}$ & $\begin{array}{c}1.69 \\
(0.78-3.64)\end{array}$ & $\begin{array}{c}0.57 \\
(0.23-1.38)\end{array}$ & $\begin{array}{c}0.40 \\
(0.06-2.82)\end{array}$ & $\begin{array}{c}0.61 \\
(0.44-0.85)\end{array}$ & $\begin{array}{c}0.67 \\
(0.38-1.20)\end{array}$ \\
\hline $\begin{array}{l}\text { Immigrant from other } \\
\text { country }\end{array}$ & $\begin{array}{c}1.29 \\
(1.00-1.68)\end{array}$ & $\begin{array}{c}0.97 \\
(0.60-1.57)\end{array}$ & $\begin{array}{c}0.69 \\
(0.46-1.03)\end{array}$ & $\begin{array}{c}0.68 \\
(0.28-1.69)\end{array}$ & $\begin{array}{c}0.71 \\
(0.59-0.84)\end{array}$ & $\begin{array}{c}0.66 \\
(0.49-0.90)\end{array}$ \\
\hline Canadian born (ref) & $1.00^{b}$ & 1.00 & 1.00 & 1.00 & 1.00 & 1.00 \\
\hline Mental health diagnosis & $\begin{array}{c}0.81 \\
(0.71-0.93)^{b}\end{array}$ & $\begin{array}{c}0.71 \\
(0.58-0.87)^{b}\end{array}$ & $\begin{array}{c}0.93 \\
(0.75-1.15)\end{array}$ & $\begin{array}{c}1.23 \\
(0.77-1.99)\end{array}$ & $\begin{array}{c}1.37 \\
(1.26-1.50)^{b}\end{array}$ & $\begin{array}{c}1.28 \\
(1.12-1.46)^{b}\end{array}$ \\
\hline \multicolumn{7}{|l|}{ Comorbidity: ADG category } \\
\hline High & $\begin{array}{c}0.69 \\
(0.59-0.82)\end{array}$ & $\begin{array}{c}1.55 \\
(1.19-2.03)\end{array}$ & $\begin{array}{c}1.33 \\
(1.01-1.73)\end{array}$ & $\begin{array}{c}1.84 \\
(0.97-2.48)\end{array}$ & $\begin{array}{c}3.31 \\
(2.95-3.71)^{b}\end{array}$ & $\begin{array}{c}3.45 \\
(2.90-4.11)\end{array}$ \\
\hline Medium & $\begin{array}{c}0.88 \\
(0.75-1.02)\end{array}$ & $\begin{array}{c}1.54 \\
(1.23-1.92)\end{array}$ & $\begin{array}{c}1.62 \\
(1.27-2.08)\end{array}$ & $\begin{array}{c}2.31 \\
(1.27-4.21)\end{array}$ & $\begin{array}{c}1.59 \\
(1.43-1.76)\end{array}$ & $\begin{array}{c}1.60 \\
(1.35-1.91)\end{array}$ \\
\hline Low (ref) & $1.00^{\mathrm{b}}$ & $1.00^{\mathrm{b}}$ & $1.00^{\mathrm{b}}$ & $1.00^{\mathrm{b}}$ & 1.00 & $1.00^{\mathrm{b}}$ \\
\hline \multicolumn{7}{|c|}{$\begin{array}{l}\text { ADG = aggregated diagnosis group; } A O R=\text { adjusted odds ratio; } A R T=\text { antiretroviral therapy; } E D=\text { emergency department; } F P=\text { family physician; ref }=\text { reference } \\
\text { group. }\end{array}$} \\
\hline \multicolumn{7}{|c|}{$\begin{array}{l}\text { a Adjusted for all listed patient covariates and interaction ter } \\
\text { b Statistically significant ( } P \text { for overall test of effect }<.05) \text {. } \\
\text { ' Family physician experience. } \\
\text { d } P=.06 \text {. }\end{array}$} \\
\hline \multicolumn{7}{|c|}{ Note: Analyses account for family physician cluster. } \\
\hline
\end{tabular}

from physician HIV experience, and mainly concluded that both are important for HIV-specific outcomes. Most of the studies in this review were conducted early in the ART era, and we were surprised to see the persistence of such a strong association between both specialty and HIV experience in our study, given the improved tolerability and decreased complexity of newer ART regimens. More recent work has shown that generalists with either HIV experience or formalized specialist decision support perform equally as well as HIV specialists for ART management. ${ }^{7,21,37}$ Physicians in our study practiced in a variety of settings, and potentially reflect a more real-world experience of care delivery to people with HIV.

We have previously demonstrated that patients cared for with models in which specialist physicians managed the majority of HIV care had lower rates of cancer screening. ${ }^{24}$ As hypothesized, in this study, we found that family physician HIV experience did not influence these primary care outcomes. Cancer screening rates were overall lower than those reported in the general Ontario population, ${ }^{38}$ a trend seen in other studies comparing screening among those with and without HIV. ${ }^{13,39}$ Furthermore, outcomes related to primary care access, such as emergency department visits and hospital admissions, were not influenced by family physician HIV experience.
There are several limitations to our work. First, our assessment of ART prescribing was restricted to patients eligible for publicly funded drug benefits, ascertained based on age older than 65 years or receipt of any drug prescription during the study period. Receipt of 1 or more ART prescriptions, however, may not reflect whether patients obtained the medications from their pharmacies, adhered to them as prescribed, or both. Second, unmeasured indicators of HIV severity may contribute to residual confounding of our findings. Third, patients cared for by family physicians with the least HIV experience were more likely to be women, to live in nonurban settings, and to have higher comorbidity, which may affect access to care or competing priorities that could contribute to lower receipt of ART. Fourth, patients seeing family physicians with higher HIV experience had slightly more outpatient visits, which may reflect improved retention and continuity associated with improved ART prescribing. Finally, the administrative databases we used do not capture care delivered outside of the provincial health insurance plan, such as in CHCs (where about $1 \%$ of the Ontario population receives primary care ${ }^{26}$ ), and to those federally insured, such as refugee claimants and some Aboriginal populations.

Our results have important implications for understanding which models of care delivery adequately 
Figure 2. Adjusted prevalence of receipt of ART among eligible patients by typology and family physician HIV experience.

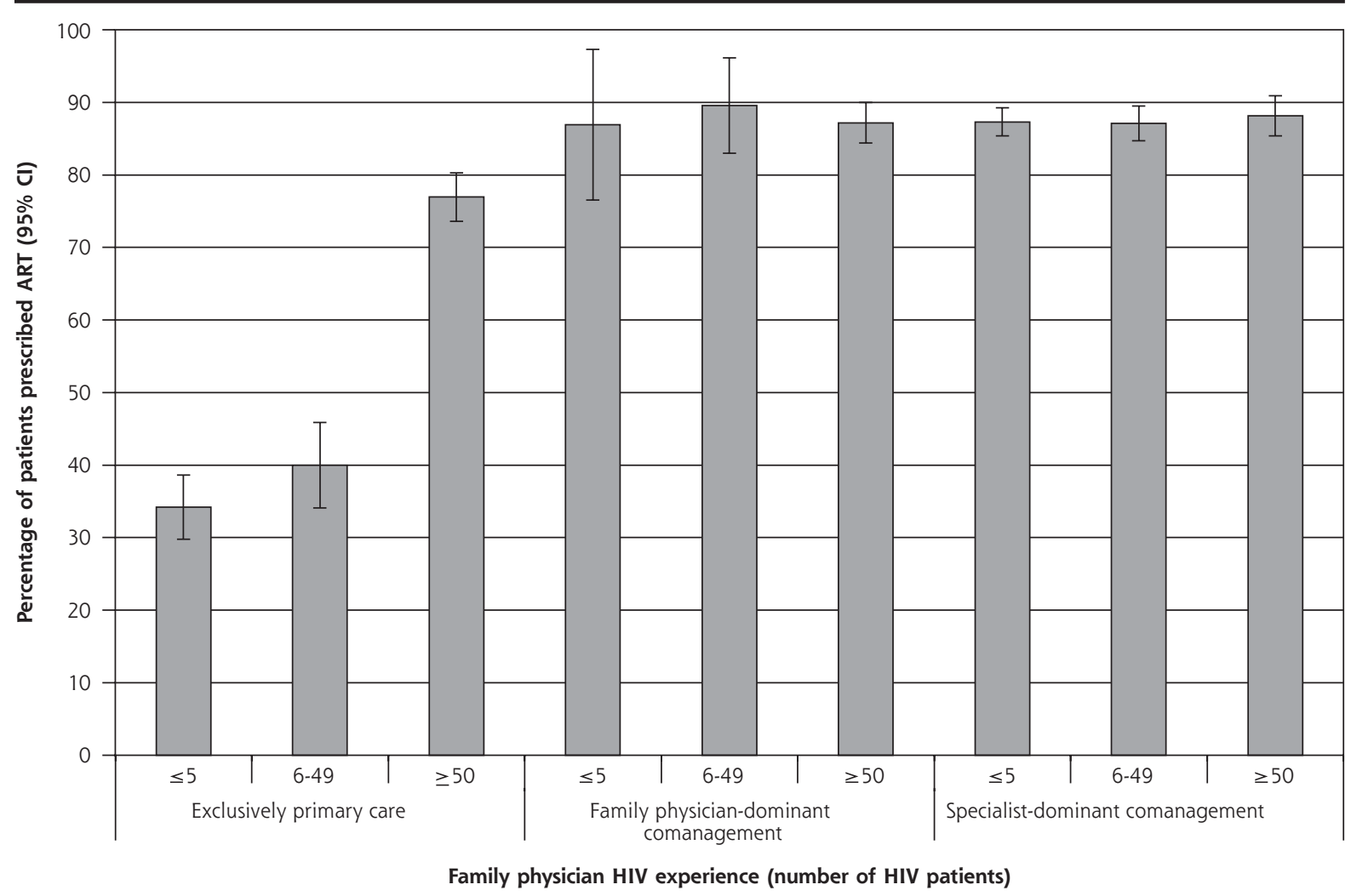

ART = antiretroviral therapy; HIV = human immunodeficiency virus

Notes: Percentage of patients with at least 1 ART prescription during study period, adjusted for all patient covariates and accounting for clustering by family physician. Error bars represent $95 \% \mathrm{Cls}$

Table 3. Mean Quality Indicators for Each Model of Care Delivery and Family Physician Level of HIV Experience

\begin{tabular}{|c|c|c|c|c|c|}
\hline $\begin{array}{l}\text { Model of Care Delivery } \\
\text { and Family Physician } \\
\text { HIV Experience }\end{array}$ & $\begin{array}{l}\text { Colorectal Cancer } \\
\text { Screening } \\
\%(95 \% \mathrm{Cl})\end{array}$ & $\begin{array}{l}\text { Cervical Cancer } \\
\text { Screening } \\
\%(95 \% \mathrm{Cl})\end{array}$ & $\begin{array}{l}\text { Breast Cancer } \\
\text { Screening } \\
\%(95 \% \mathrm{Cl})\end{array}$ & $\begin{array}{l}\text { Any ED Visit } \\
\%(95 \% \mathrm{Cl})\end{array}$ & $\begin{array}{l}\text { Any Hospital } \\
\text { Admission } \\
\%(95 \% \mathrm{Cl})\end{array}$ \\
\hline \multicolumn{6}{|l|}{ Exclusively primary care } \\
\hline$\leq 5$ HIV patients & $39(32-47)$ & $56(50-62)$ & $66(56-77)$ & $33(30-35)$ & $7(6-9)$ \\
\hline 6-49 HIV patients & $34(25-43)$ & $41(32-50)$ & $61(44-79)$ & $32(28-36)$ & $9(7-11)$ \\
\hline$\geq 50$ HIV patients & $43(36-50)$ & $54(45-63)$ & $49(22-68)$ & $29(27-32)$ & $6(5-7)$ \\
\hline \multicolumn{6}{|l|}{$\begin{array}{l}\text { Family physician-dominant } \\
\text { comanagement }\end{array}$} \\
\hline$\leq 5$ HIV patients & $44(14-75)$ & $59(27-91)$ & $37(0-90)$ & $26(13-39)$ & $18(7-28)$ \\
\hline 6-49 HIV patients & $43(22-63)$ & $26(4-49)$ & $35(0-81)$ & $31(22-39)$ & $13(7-19)$ \\
\hline$\geq 50$ HIV patients & $49(40-58)$ & $48(36-59)$ & $79(60-97)$ & $32(28-35)$ & $13(11-15)$ \\
\hline \multicolumn{6}{|l|}{$\begin{array}{l}\text { Specialist-dominant } \\
\text { comanagement }\end{array}$} \\
\hline$\leq 5$ HIV patients & $30(25-35)$ & $47(42-52)$ & $46(35-56)$ & $33(30-35)$ & $10(9-11)$ \\
\hline 6-49 HIV patients & $25(19-31)$ & $39(33-46)$ & $61(47-76)$ & $34(31-37)$ & $9(7-11)$ \\
\hline$\geq 50$ HIV patients & $40(31-49)$ & $49(35-57)$ & $31(7-56)$ & $32(28-35)$ & $10(8-12)$ \\
\hline
\end{tabular}

HIV = human immunodeficiency virus; ED = emergency department.

Note: Analyses are adjusted for patient age, sex, income quintile, rurality, immigrant status, aggregated diagnosis group category, presence of a mental health condition, and experience category of the family physician, and account for clustering by family physician. 
integrate the strengths of both specialists and family physicians in optimizing access to and quality of comprehensive care for people living with HIV. ${ }^{19,40}$ Sixteen percent of HIV patients in Ontario receive care exclusively from family physicians with lower HIV experience; our results suggest potential disparities in ART among these patients. Because we also found that this influence of family physician HIV experience is mitigated by having an HIV specialist within their model of care, in order to ensure adequate ART prescribing, care delivery models for people with HIV should include either an HIV specialist or a family physician with considerable HIV experience. We recommend that such access to HIV expertise, whether through direct contact with HIV specialists or through strategies that give decision support to family physicians, be provided within a collaborative model that allows patients to retain continuity with their family physicians to ensure a comprehensive approach to care delivery. ${ }^{19,40-42}$

\section{To read or post commentaries in response to this article, see it} online at http://www.annfammed.org/content/13/5/436.

Key words: human immunodeficiency virus; primary care; specialists; chronic disease; comorbidity; health services delivery

Submitted January 8, 2015; submitted, revised, May 11, 2015; accepted June 9, 2015.

Funding support: This research was funded in part by the Ontario Ministry of Health and Long-term Care (MOHLTC). This study was supported by the Institute for Clinical Evaluative Sciences (ICES), which is funded by an annual grant from MOHLTC.

Disclaimer: The opinions, results, and conclusions reported in this article are those of the authors and are independent from the funding sources. No endorsement by ICES or the Ontario MOHLTC is intended or should be inferred. Claire Kendall holds a Canadian Institutes for Health Research (CIHR) Fellowship in the Area of Health Services/Population Health HIVIAIDS Research. Richard Glazier is supported as a Clinician Scientist in the Department of Family and Community Medicine at the University of Toronto and at St Michael's Hospital.

Previous presentations: The corresponding author presented an earlier version of these analyses at the North American Primary Care Research Group Annual Meeting; November 21-25, 2014; New York City, New York.

Acknowledgments: We are grateful to Lisa Boucher for her assistance in preparing this manuscript.

\section{References}

1. Handford C, Tynan A-M, Rackal J, Glazier R. Setting and organization of care for persons living with HIV/AIDS. Cochrane Database Syst Rev. 2006;(3):CD004348.

2. Handford CD, Rackal JM, Tynan A-MM, Rzeznikiewiz D, Glazier RH. The association of hospital, clinic and provider volume with HIV/ AIDS care and mortality: systematic review and meta-analysis. AIDS Care. 2012;24(3):267-282
3. Rackal JM, Tynan A-MM, Handford CD, Rzeznikiewiz D, Agha A, Glazier R. Provider training and experience for people living with HIV/AIDS. Cochrane Database Syst Rev. 2011;(6):CD003938.

4. Kitahata MM, Van Rompaey SE, Dillingham PW, et al. Primary care delivery is associated with greater physician experience and improved survival among persons with AIDS. J Gen Intern Med. 2003;18(2):95-103.

5. Landon BE, Wilson IB, Cohn SE, et al. Physician specialization and antiretroviral therapy for HIV. J Gen Intern Med. 2003;18(4):233-241.

6. Wilson IB, Landon BE, Hirschhorn LR, et al. Quality of HIV care provided by nurse practitioners, physician assistants, and physicians. Ann Intern Med. 2005;143(10):729-736.

7. Chu C, Umanski G, Blank A, Grossberg R, Selwyn PA. HIVinfected patients and treatment outcomes: an equivalence study of community-located, primary care-based HIV treatment vs. hospital-based specialty care in the Bronx, New York. AIDS Care. 2010;22(12):1522-1529.

8. Koethe JR, Moore RD, Wagner KR. Physician specialization and women's primary care services in an urban HIV clinic. AIDS Patient Care STDS. 2008;22(5):373-380.

9. Horberg MA, Hurley LB, Towner WJ, et al. Determination of optimized multidisciplinary care team for maximal antiretroviral therapy adherence. J Acquir Immune Defic Syndr. 2012;60(2):183-190.

10. Fultz SL, Goulet JL, Weissman S, et al. Differences between infectious diseases-certified physicians and general medicine-certified physicians in the level of comfort with providing primary care to patients. Clin Infect Dis. 2005;41(5):738-743.

11. Duffus WA, Barragan M, Metsch L, et al. Antiretroviral Treatment and Access Studies Study Group. Effect of physician specialty on counseling practices and medical referral patterns among physicians caring for disadvantaged human immunodeficiency virus-infected populations. Clin Infect Dis. 2003;36(12):1577-1584.

12. Sheth AN, Moore RD, Gebo KA. Provision of general and HIVspecific health maintenance in middle aged and older patients in an urban HIV clinic. AIDS Patient Care STDS. 2006;20(5):318-325.

13. Reinhold JP, Moon M, Tenner CT, Poles MA, Bini EJ. Colorectal cancer screening in HIV-infected patients 50 years of age and older: missed opportunities for prevention. Am J Gastroenterol. 2005;100 (8):1805-1812.

14. Leece P, Kendall C, Touchie C, Pottie K, Angel JB, Jaffey J. Cervical cancer screening among HIV-positive women. Retrospective cohort study from a tertiary care HIV clinic. Can Fam Physician. 2010;56 (12):e425-e431.

15. Aberg JA, Gallant JE, Ghanem KG, Emmanuel P, Zingman BS, Horberg MA; Infectious Diseases Society of America. Primary care guidelines for the management of persons infected with HIV: 2013 update by the HIV Medicine Association of the Infectious Diseases Society of America. Clin Infect Dis. 2014;58(1):e1-e34.

16. Justice AC. HIV and aging: time for a new paradigm. Curr HIVIAIDS Rep. 2010;7(2):69-76.

17. Kendall CE, Guenter D. Time to deliver on HIV care. Can Fam Physician. 2006;52:1449-1450.

18. Cahill S, Darnell B, Guidry JA, et al. Growing Older With the Epidemic: HIV and Aging. New York, NY: Gay Men's Health Crisis; 2010.

19. Chu C, Selwyn PA. An epidemic in evolution: the need for new models of HIV care in the chronic disease era. J Urban Health. 2011; 88(3):556-566.

20. Page J, Weber R, Somaini B, Nöstlinger C, Donath $K$, Jaccard $R_{i}$ SESAM Study Group. Quality of generalist vs. specialty care for people with HIV on antiretroviral treatment: a prospective cohort study. HIV Med. 2003:4(3):276-286.

21. Kerr CA, Neeman N, Davis RB, et al. HIV quality of care assessment at an academic hospital: outcomes and lessons learned. Am J Med Qual. 2012;27(4):321-328. 
22. Forrest CB. A typology of specialists' clinical roles. Arch Intern Med. 2009;169(11):1062-1068.

23. Kendall CE, Younger J, Manuel DG, Glazier RH, Hogg WE, Taljaard $M$. Health administrative data can be used to define a shared care typology for people with HIV. J Clin Epidemiol. 2015;508954356(15)00103-1. http://www.jclinepi.com/article/S0895-4356(15) 00103-1/fulltext. Published Feb 20, 2015. Accessed Aug 10, 2015.

24. Kendall CE, Manuel DG, Younger J, Hogg WE, Glazier RH, Taljaard M. A population-based study comparing patterns of care delivery on the quality of care for persons living with HIV in Ontario. BMJ Open. 2015; 5(5):e007428.

25. Antoniou T, Zagorski B, Loutfy MR, Strike C, Glazier RH. Validation of case-finding algorithms derived from administrative data for identifying adults living with human immunodeficiency virus infection. PLoS One. 2011;6(6):e21748.

26. Glazier R, Zagorski B, Rayner J. Comparison of Primary Care Models in Ontario by Demographics, Case Mix and Emergency Department Use, 2008/09 to 2009/10. ICES Investigative Report. Toronto, Ontario: Institute for Clinical Evaluative Sciences; 2012.

27. Kralj B. Measuring "rurality" for purposes of health care planning: an empirical measure for Ontario. Ont Med Rev. 2000;67(9):33-52.

28. Public Health Agency of Canada. HIV/AIDS Epi Update: National HIV Prevalence and Incidence Estimates in Canada for 2008. Ottawa, Ontario: Public Health Agency of Canada; 2010.

29. Remis RS, Swantee C, Liu J. HIV/AIDS in Ontario: Preliminary Report, 2010. Toronto, Ontario: Ontario HIV Epidemiologic Monitoring Unit; 2012.

30. Rebeiro P, Althoff KN, Buchacz K, et al; North American AIDS Cohort Collaboration on Research and Design. Retention among North American HIV-infected persons in clinical care, 2000-2008. J Acquir Immune Defic Syndr. 2013;62(3):356-362.

31. Krentz HB, Gill MJ. Comparison of healthcare costs between local and immigrant HIV populations living in Southern Alberta, Canada. Health Policy. 2011;103(2-3):124-129.

32. Dombrowski JC, Kent JB, Buskin SE, Stekler JD, Golden MR. Population-based metrics for the timing of HIV diagnosis, engagement in HIV care, and virologic suppression. AIDS. 2012;26(1):77-86.
33. Huntley AL, Johnson R, Purdy S, Valderas JM, Salisbury C. Measures of multimorbidity and morbidity burden for use in primary care and community settings: a systematic review and guide. Ann Fam Med. 2012;10(2):134-141.

34. The Johns Hopkins University. The Johns Hopkins Adjusted Clinical Groups (ACG) System. 1997. http://www.acg.jhsph.org. Accessed Aug 10, 2015.

35. Steele LS, Glazier RH, Lin E, Evans M. Using administrative data to measure ambulatory mental health service provision in primary care. Med Care. 2004;42(10):960-965.

36. Jaakkimainen L, Guttmann A, Klein-Geltink JE, et al. Indicators of primary care based on administrative data. In: Jaakkimainen $L$, Klein-Geltink J, Leong A, Maaten S, Schultz SE, et al, eds. Primary Care in Ontario: ICES Atlas. Toronto, Canada: Institute for Clinical Evaluative Sciences; 2006:207-249.

37. Chu C, Heo M, Peshansky A, et al. Comparable sustained virologic suppression between community- and academic-based HIV care settings. J Am Board Fam Med. 2015;28(1):72-81.

38. Kiran T, Wilton AS, Moineddin R, Paszat L, Glazier RH. Effect of payment incentives on cancer screening in Ontario primary care. Ann Fam Med. 2014;12(4):317-323.

39. Rahangdale L, Sarnquist C, Yavari A, Blumenthal P, Israelski D. Frequency of cervical cancer and breast cancer screening in HIVinfected women in a county-based HIV clinic in the Western United States. J Womens Health (Larchmt). 2010;19(4):709-712.

40. Boyd CM, Lucas GM. Patient-centered care for people living with multimorbidity. Curr Opin HIV AIDS. 2014;9(4):419-427.

41. Pasricha A, Deinstadt RT, Moher D, Killoran A, Rourke SB, Kendall CE. Chronic Care Model Decision Support and Clinical Information Systems interventions for people living with HIV: a systematic review. J Gen Intern Med. 2013;28(1):127-135.

42. Mugavero MJ, Norton WE, Saag MS. Health care system and policy factors influencing engagement in HIV medical care: piecing together the fragments of a fractured health care delivery system. Clin Infect Dis. 2011;52(Suppl 2):S238-S246. 